\title{
UN CASO DIFFICILE NEL TESTO DELLA FISICA DI ARISTOTELE (216A29-31) NEL COMMENTARIO DI GIOVANNI FILOPONO*
}

ABSTRACT: I commenti neoplatonici ad Aristotele, oltre a dare indicazioni interpretative su questioni di contenuto, possono non solo far riflettere ma anche dare importanti indicazioni sul testo aristotelico e sulla traditio textus. In questo saggio verrà illustrato un caso particolarmente significativo, contenuto nel commento alla Fisica scritto da Giovanni Filopono (prima metà del VI secolo): in Phys. 216a2931, infatti, occorre una lectio ( $\sigma v v i ́ \sigma \tau \alpha \sigma \theta \alpha \mathrm{l}$ ) diversa dal testo canonico di Aristotele attestato dai manoscritti ( $\mu \varepsilon \theta i ́ \sigma \tau \alpha \sigma \theta \alpha \imath)$. La competenza filologica di Filopono avvalora particolarmente questa lectio che risulta non aver avuto nell'antichità adeguate testimonianze; infatti, i manoscritti medievali continuano una tradizione diversa. Il caso esaminato intende riportare l'attenzione sull'importanza che la produzione ipomnematica tardoantica può offrire nel valutare, almeno in casi particolari, quale testo dello Stagirita circolasse prima che si costituisse una vulgata aristotelica, quella data dai codici medievali.

KEY WORDS: Aristotele, Giovanni Filopono, testo, Fisica, commento

\section{INTRODUZIONE}

I commentatori neoplatonici tardoantichi ad Aristotele stanno conoscendo una primavera di studio negli ultimi anni a partire soprattutto

Ringrazio fervidamente i due referee anonimi per le preziose indicazioni. 
dalla notoria serie The Ancient Commentators on Aristotle iniziata e diretta da Richard Sorabji; tali commentatori, infatti, apportano un contributo decisivo sotto il rispetto dell'interpretazione speculativa dello Stagirita e un contributo altrettanto rilevante potrà però essere offerto dal medesimo corpus ipomnematico anche sotto un altro punto di vista - non raramente negletto, malgrado alcuni recenti contributi ${ }^{1}-$, cioè la constitutio textus di Aristotele: i commentatori tardoantichi possono far luce o almeno far riflettere sullo stato del testo aristotelico prima della tradizione manoscritta medievale, da cui de facto dipende 1'Aristotele che leggiamo ${ }^{2}$. Rilevante è soprattutto il caso di Giovanni Filopono, autore noto in antico non solo, nello specifico, per la fine competenza filosofica sulla pagina aristotelica ma anche per la sua acribia filologica, più in generale. Di qui acquista particolare interesse considerare alcune lezioni testimoniate solo in Filopono, puntualmente riferite in nota ad es. dal Ross ma finora non vagliate nelle loro ragioni specifiche, così da portare un raggio di luce sulle condizioni del testo che di Aristotele veniva letto in VI secolo. Questo non significherà ipso facto correggere il textus receptus ma, con maggior moderazione, permette almeno di riflettere sulle condizioni che hanno portato alla sua genesi e di affrontare auspicabilmente con maggior consapevolezza le questioni filosofiche che la lettera reca con sé.

\section{IL CASO DI PHYS. 216A29-31: TESTO ED ESEGESI}

In questo quadro, tra le questioni che vanno emergendo nello studio del commento alla Fisica redatto da Filopono è centrale la domanda circa il testo aristotelico che l'alessandrino avesse ad antigrafo e da cui estraesse

\footnotetext{
$1 \quad$ A livello più generale cfr. Golitsis 2008 e Rashed 2008 mentre, nello specifico della Fisica aristotelica, si rinvia al capitolo di Marwan Rashed intitolato Fragments inconnus du commentaire à la Physique de Jean Philopon in Rashed 2016: 755-777 e Rescigno 2017, oltre a Sjoerd Hasper 2020.

2 Sull'apporto dei commentatori tardoantichi alla valutazione, specificamente, del testo della Fisica aristotelica, cfr. già Diels 1882 e, in anni recenti, Ross 1979 (soprattutto il cap. III, in cui si sottolinea la specificità delle uariae lectiones dei commentatori neoplatonici di contro alla tradizione medievale che si mostra spesso fluttuante e alquanto indocile a essere sussunta in una rigida stemmatica).
} 
i lemmi che commenta. Si intende qui illustrare il caso rilevante costituito da Phys. 216a29ss (un luogo che occorre nelle more di una lunga sezione pertinente alla cinetica nel vuoto). Vale riferire anzitutto la pericope in esame:

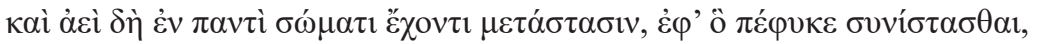

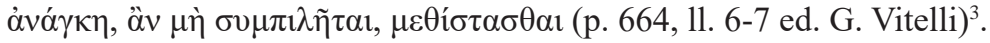

Intanto è opportuno mettere a fuoco che, nel commento di Filopono, questa uaria lectio occorre due volte: alle citate 11. 6-7, infatti, essa figura una prima volta, nell'interno del lemma che l'edizione del commentatore riporta come suo uso prima di intraprendere le osservazioni pertinenti alla pericope in indagine. Se è vero che a oggi manca uno studio che esamini direttamente la condizione dei lemmi nelle opere ipomnematiche ad Aristotele ${ }^{4}$ e se è parimenti vero che l'autenticità dei medesimi lemmi non è da escludersi ma nemmeno è acriticamente riconducibile alla mano del medesimo Filopono, assume particolare rilievo la seconda occorrenza, capace qual è di revocare ogni dubbio circa il testo che Filopono leggeva. Scrive infatti poco oltre:

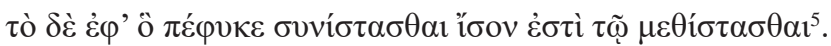

Tale seconda occorrenza cassa ogni possibilità di reticenza in merito, poiché mostra ostensibilmente che Filopono doveva avere davanti a sé un testimone con $\sigma v v i ́ \sigma \tau \alpha \sigma \theta \alpha \iota$ e il testo riportato nel lemma, quand'anche non rimontasse alla mano del commentatore, certo in modo autoschediastico riprende il testo (ormai attestato, dunque, utrobique) che il maestro aveva citato nel corso stesso del suo commento.

Si tratta di un luogo che indaga una questione molto circoscritta e forse minore, qual è la natura del movimento di aria, acqua o fuoco nel momento in cui un corpo estraneo - preferibilmente di forma

3 L'edizione di riferimento è Ioannis Philoponi in Aristotelis Physicorum libros quinque posteriores commentaria, Berolini 1888, (qui pp. 664, 6-665, 15).

4 Reciso circa la loro inattendibilità (almeno in riferimento alla Fisica) è ancora Carteron: «les lemmes de Philopon sont inutilisable» (nell'introduzione ad Arist., Physique (I-IV), Paris 1990 (19261), 5, n. 1.

$5 \quad$ Philop., In Phys. 665, 12-13 (ed. Vitelli). 
cubica - venga inserito in ciascuno di essi ${ }^{6}$. Come si nota, la lezione di Aristotele prodotta e commentata da Filopono presenta in primo luogo l'infinito $\sigma v v i ́ \sigma \tau \alpha \sigma \theta \alpha \mathrm{l}$ e, subito dopo, l'infinito $\mu \varepsilon \theta$ í $\tau \alpha \sigma \theta \alpha \mathrm{l}$, quando invece la vulgata aristotelica è concorde nello scrivere in ambedue le posizioni $\mu \varepsilon \theta i ́ \sigma \tau \alpha \sigma \theta \alpha$. Una volta aver riconosciuto che si tratta sì di due corradicali distinti per il solo prefisso, va osservato tuttavia che il senso delle due composizioni verbali, calate nel contesto, risulta essere non identico, sia ovviamente a livello lessicale sia in ispecie a livello sintattico; infatti nel caso di $\sigma u v i ́ \sigma \tau \alpha \sigma \theta \alpha$ interviene un'accezione in prevalenza statica ('stare-con; consistere; constare' e tale è, in ogni caso, il valore predominante in Aristotele) mentre in $\mu \varepsilon \theta i ́ \sigma \tau \alpha \sigma \theta \alpha$ il prefisso $\mu \varepsilon \tau \alpha$ - conferisce un valore prevalentemente dinamico al senso in sé statico del verbo í $\sigma \alpha \mu \alpha$, 'stare in piedi/star fermo' (mentre $\mu \varepsilon \theta i ́ \sigma \tau \alpha \sigma \theta \alpha$ l varrà quindi 'spostarsi'): posto che l'accezione di $\sigma v v i ́ \sigma \tau \alpha \sigma \theta \alpha$ lè strettamente vincolata nella prima occorrenza al senso sostanzialmente di movimento espresso dal precedente $\dot{\varepsilon} \varphi$ ' ő (che mal si concilia con un'idea verbale principalmente statica), è facile comprendere che è in gioco non solo una variante testuale ma anche l'aspetto esegetico che ne dipende.

Prima di affrontare nel dettaglio i contenuti del passo, occorre speci-

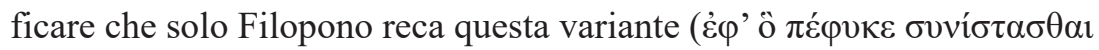

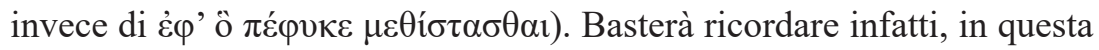
sede, che i codd. EFHIJ scrivono concordemente due volte $\mu \varepsilon \theta i ́ \sigma \tau \alpha \sigma \theta \alpha$ l (i citati codici EFIJ - che recano l'intero testo della Fisica, a parte il libro VII, presente solo per escerpti - oscillano tra l'inizio del X secolo e il XIV secolo: E (Par. Gr. 1853) è dell'inizio del X mentre F (Laur.

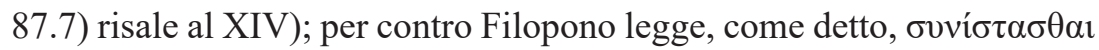
in luogo del primo $\mu \varepsilon \theta i ́ \sigma \tau \alpha \sigma \theta \alpha$ s (accompagnato in questo dal solo riscontro di $\mathbf{J}^{\mathbf{1}}$ supra lineam). Stante la diuturna competenza che Giovanni Filopono poteva vantare sulla lettera di Aristotele, maturata in anni di esegesi, stante la sua citata perizia grammaticale (questi era infatti noto come ó $\gamma \rho \alpha \mu \mu \alpha \tau$ có $\zeta)^{7}$ e stante altresì la qualità della sua produzione espressamente filologica, senza contare poi che in molti passi - e anche

\footnotetext{
6 Per un quadro di riferimento generale sulla teoria aristotelica del moto naturale in connessione con i luoghi naturali, si rimanda almeno ad Algra 1995: 195-222.

7 Più in generale, anche prescindendo almeno per il momento dalla competenza filologica di Filopono, va notato che i commentatori di VI secolo esibiscono un'attenzione e una consapevolezza grandi per la lezione del testo tanto che Simplicio arriva
} 
nella nostra pericope, come vedremo - egli si pronuncia recta uia su questioni testuali ${ }^{8}$-, è evidente che il caso richiede di essere indagato sia per intendere qualcosa del testo aristotelico su cui il commentatore alessandrino lavorava sia per intendere la filigrana della lettera dello Stagirita, in cui ogni flebile variazione testuale può essere latrice di variazioni anche sensibili in ordine teoretico.

Giova dunque fare tre osservazioni, prima di proseguire: I) non ci sono dubbi che Filopono leggesse $\sigma v v i ́ \sigma \tau \alpha \sigma \theta \alpha \imath$ (questa lezione compare non solo nel lemma, che può essere redazionale, ma anche nel corso del testo); II) il senso di $\sigma v v i ́ \sigma \tau \alpha \sigma \theta \alpha \iota$ è percepito come pari ('̌́ov) a quello di $\mu \varepsilon \theta i ́ \sigma \tau \alpha \sigma \theta \alpha \mathrm{l}$ : pur non essendoci perfetta sovrapponibilità fra i testi a seconda della variante impiegata, vige tuttavia un sostanziale accordo sul senso di fondo; III) in ultimo, la correzione sopralineare di $\mathbf{J}^{\mathbf{1}}$ può essere un'integrazione apportata dal correttore proprio attingendo a Filopono, tanto più se si considera l'autorità che questi ben presto si era procurato. In ogni caso, se anche così non fosse e $\mathbf{J}^{\mathbf{1}}$ non fosse influenzato da Filopono, la variante messa a testo da quest'ultimo sarebbe allora non più unica ma comunque rarissima - e quindi l'economia complessiva della questione cambierebbe poco.

Sull'orizzonte di questi riscontri, deve essere sempre tenuto presente che Filopono conosce solo il testo con $\sigma v v i ́ \sigma \tau \alpha \sigma \theta \alpha$, non riportando questa come una variante (minore o no) di $\mu \varepsilon \theta i ́ \sigma \tau \alpha \sigma \theta \alpha$. Infatti, quando la tradizione manoscritta gli sottoponeva delle varianti, Filopono usa almeno citare la variante non accolta. Tale rilievo si inserisce perfettamente nello zelo filologico di Filopono, alla cui meticolosità testuale non sarebbe sfuggita l'occasione di riferire l'eventuale natura di questa lezione come variante di un'altra, più o meno testimoniata, se solo gli fosse stata nota. A questo proposito sarà sufficiente menzionare quanto si

addirittura a correggere Temistio su questioni testuali, in una querelle filologica: cfr. Henri Carteron nell'introduzione ad Arist., Physique (I-IV), Paris 1990 (19261).

Si può ricordare, semel pro omnibus, il caso di Philop., In Phys. 894, 5.10-11 (ed.

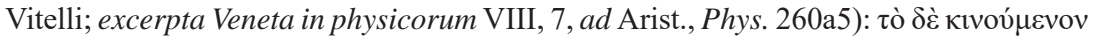

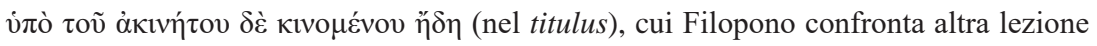

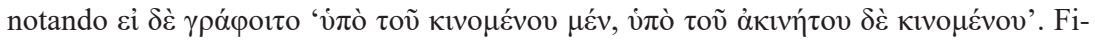
lopono affianca all'attenzione per la lettera aristotelica anche grande moderazione, per non eccedere in sottigliezze: così Philop., In Phys. 900, 12 (ed. Vitelli; excerpta Veneta

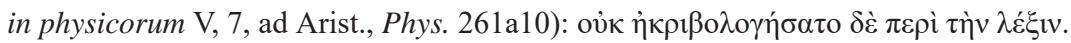


legge nella nostra medesima pericope, poco sotto, allorché si dice di un cubo messo dentro l'aria:

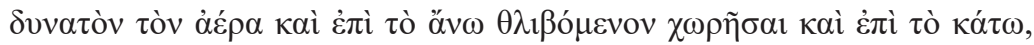

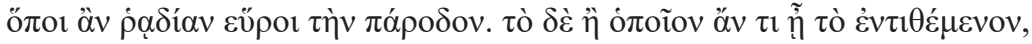

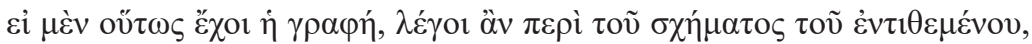

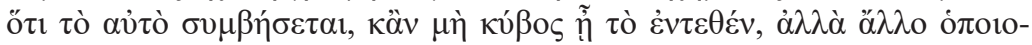
võ๊v $\sigma \chi \tilde{\eta} \mu \alpha^{9}$.

L'attenzione testuale di Filopono è perspicua: trattando dello spostamento causato dal cubo nell'aria, viene osservato che l'aria ha due possibilità, giacché essa avanza comprimendosi o verso l'alto o verso il basso, a seconda di dove trovi più facile l'accesso. La condizione che determina e governa la direzione di questo spostamento meccanicamente indotto viene quindi fatta oggetto di un rilievo filologico da parte di Filopono:

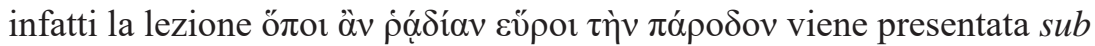
judice, perché come prima ipotesi il grammatico avanza la lectio alternativa ó $\pi$ oĩov $\alpha$ ó , ove l'oscillazione deve essere ascritta a uno scambio alpha/omicron e a una conseguente diversa distinctio uerborum. Prescindendo in questa sede da osservazioni circa la co-testualità e le interrelazioni con quanto precede e segue (sia sintatticamente sia a livello di referenzialità testuale: l'intorno del testo), vanno fatte, ancora, almeno tre osservazioni: I) la lezione ó poiché Filopono direbbe che lo spostamento dell'aria avviene quale che sia la forma dell'oggetto immessovi, non solo se esso è un cubo (la

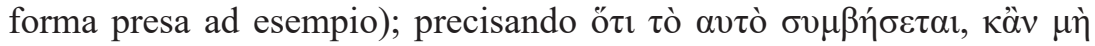

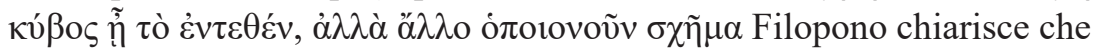
la forma cubica non influisce in modo determinante sul fenomeno sotto

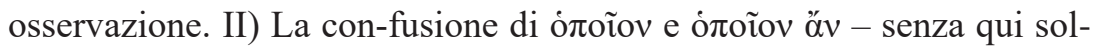
levare altre obiezioni, come la presenza di ớv in ó $\pi$ oĩov $\alpha$ óv - è plausibile a partire dallo scambio soprallodato tra alpha e omicron, il che presuppone che Filopono leggesse un testimone vergato in minuscola, dove il tratteggio circolare di alpha poteva dare adito a un accostamento grafico con il modulo di omicron (alla qual cosa osterebbe invece il maiuscolo). Si tratterebbe di un fatto grafico non insolito nella koinè greco-romana e, più in generale, tale episodio di minuscola libraria si lascia ricondurre

$9 \quad$ Philop., In Phys. 664, 29-665, 4 (ed. Vitelli). 
a una suggestione scrittoria di ascendenza cancelleresca già di III-IV secolo, destinata a imprimere il proprio stigma anche nei papiri letterari ${ }^{10}$, tenendo sempre presente che i medesimi spesso derogano de facto al carattere calligrafico e si impregnano di evidenze corsive ${ }^{11}$. III) Posto che, a seconda che si legga alpha o omicron, avverrà un fenomeno di accrezione o discrezione di $-\alpha v-$ a quanto precede, va soprattutto notato lo scrupolo con cui Filopono interviene sul punto agitato, riconoscendo che tutto dipende dal fatto che sia fededegna o meno la lezione: $\varepsilon \grave{l} \mu \dot{\varepsilon} v$

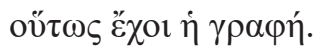

\section{APPROFONDIMENTI ESEGETICI}

Come si constata, è questo un esempio - non solo uno tra i tanti ma un esempio che si trova proprio nello stesso passo in esame - il quale dimostra la cura di Filopono verso la lezione che sceglie; di qui non è economico pensare che Filopono, in Phys. 216a29-31, fosse a conoscenza della variante $\mu \varepsilon \theta i ́ \sigma \tau \alpha \sigma \theta \alpha$ e l'abbia lasciata cadere senza neanche degnarla di attenzione, almeno en passant. Circa lo spostamento prodotto dal cubo, Filopono avanza poi anche l'ipotesi alternativa, che vedremo essere rilevante proprio al fine di intendere la uaria lectio che stiamo esaminando. Scriveva l'esegeta

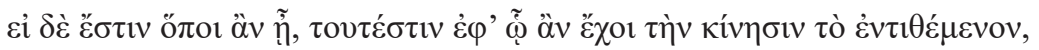

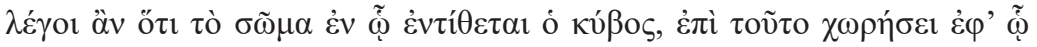

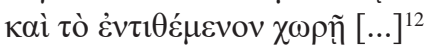

Se, allora, si legge il testo con questa variante, a essere messa a fuoco sarà non più la forma del grave bensì la direzione verso cui avanza l'oggetto posto dentro il suo contenente; ma così facendo, se si accetta ő si avverte il contrasto tra il valore di moto, direttivo, allativo, che questo avverbio relativo ha ('verso dove', non 'dove': latinamente quo, non ubi) e l'idea statica invece insita nel verbo essere cui si accompagna (ทิ) $)$ a questo punto giova riscontrare che tale contrasto non osta a che

\footnotetext{
10 Cfr. Crisci - Degni 2011: 82-87 e 96-101.

11 Messeri - Pintaudi 2000: I, 67-82, qui 81.

12 Philop., In Phys. 665, 4-6 (ed. Vitelli).
} 
Filopono consideri comunque possibile e capace di senso anche tale junctura (verbo statico più espressione di moto), in modo strutturalmente

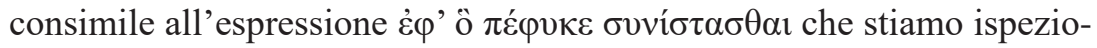
nando. Su questi elementi si incardina un tratto che passim è già emerso, cioè la lezione $\sigma v v i ́ \sigma \tau \alpha \sigma \theta \alpha$ è senz'altro lectio difficilior sotto il rispetto del senso. Circa la pericope coinvolta nel commento di Phys. 216a29-31,

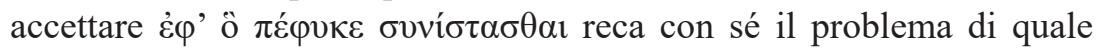
senso attribuire a $\dot{\varepsilon} \varphi$ ' ö, che non sarebbe agevole deprivare del senso di moto; il senso di moto, tuttavia, non è in prima istanza consono con

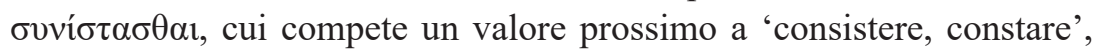
in armonia con i suoi costituenti ${ }^{13}$. In questi termini, $\mu \varepsilon \theta i ́ \sigma \tau \alpha \sigma \theta \alpha$, invece, appianerebbe eo ipso la medesima difficoltà, perché il prefisso $\mu \varepsilon \tau \alpha$ - ('dopo', donde $\mu \varepsilon \theta i ́ \sigma \tau \alpha \sigma \theta \alpha$ l come 'porsi dopo, trasferirsi') conferisce un valore di spostamento al radicale cui si applica, così da avere una valenza morfosintattica saturabile dal citato $\dot{\varepsilon} \varphi$ ' ö. Accettare di scrivere $\sigma v v i ́ \sigma \tau \alpha \sigma \theta \alpha \iota$ richiede di necessità di affrontare nella sua sottigliezza tale questione, consapevoli che il testo di Aristotele per il suo equilibrio speculativo vive (o vive anche) di queste cose sottili. Preventivamente, va guardata con molta perplessità la risposta, pur comoda ma inaccettabilmente semplificatoria, secondo cui $\sigma v v i ́ \sigma \tau \alpha \sigma \theta \alpha \iota$ sarebbe una forma di corruttela, nata dal più piano $\mu \varepsilon \theta i ́ \sigma \tau \alpha \sigma \theta \alpha$ ı per effetto di una sorta di dissimilazione dal precedente $\mu \varepsilon \tau \alpha ́ \sigma \tau \alpha \sigma ı v$ : con lo stesso metro, si sarebbe potuta determinare una dissimilazione tra il primo e il secondo $\mu \varepsilon \theta i ́ \sigma \tau \alpha \sigma \theta \alpha$, tanto da avere in successione $\mu \varepsilon \theta i ́ \sigma \tau \alpha \sigma \theta \alpha \imath$ e $\sigma v v i ́ \sigma \tau \alpha \sigma \theta \alpha$, in ordine inverso a come invece storicamente le due forme sono a noi

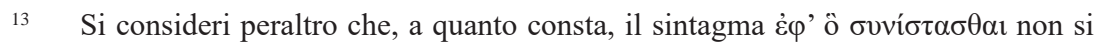
incontra altrove non solo in Aristotele ma nemmeno in alcun testo greco a noi pervenuto. Ciò depone a favore della natura di lectio difficillima del testo filoponiano (il quale quindi non poteva originarsi per semplice corruzione dal più piano $\mu \varepsilon \theta i \sigma \tau \alpha \sigma \theta \alpha \mathrm{l})$; in questa luce, proprio per l'imbarazzo che doveva presentare tale lezione, il codice $\mathbf{M}$ (Marcianus 230) doveva cercare di risolvere il problema scrivendo $\dot{\varepsilon} \varphi$ ' $\tilde{\varphi}$, pressoché banalizzando la lezione filoponiana sulla base del più diffuso nesso di $\sigma v v i ́ \sigma \tau \alpha \sigma \theta \alpha$ r costruito con غ̇ंí plus dativo. La difficoltà peraltro affiora anche dalla strana nota che

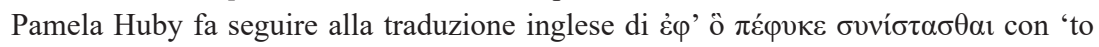
move towards its natural goal', scrivendo «the lemma has sunistanai (sic) which is supported at 665,13 by Philoponus' own sunistanai, but Aristotle's text (sic) has methistanai (sic)» per cui cfr. Philoponus 2012: ad loc., n. 159. 
testimoniate. Inoltre, resta centrale la questione di senso, giacché l'accezione di moto con $\sigma u v i ́ \sigma \tau \alpha \sigma \theta \alpha$ resta oltremodo scomoda presso lo stile aristotelico.

In quanto forma più difficile, non potrà qui essere accolto che da $\mu \varepsilon \theta i ́ \sigma \tau \alpha \sigma \theta \alpha$ si sia generato il più impegnativo $\sigma v v i ́ \sigma \tau \alpha \sigma \theta \alpha$; piuttosto, a questo livello di indagine, si potrà affermare che il più connotato $\sigma v v i ́ \sigma \tau \alpha \sigma \theta \alpha$ abbia poi conosciuto un'assimilazione - questa volta effettivamente da avanzare - così da normalizzarsi in $\mu \varepsilon \theta i \sigma \tau \alpha \sigma \theta \alpha$, restituendo un testo che scorre sintatticamente e concettualmente, senza non liquet anche se a costo di una banalizzazione. Resta da capire il senso che il luogo doveva avere nella sua versione originaria e - questione non del tutto coincidente - quale significato riuscisse ancora a percepirvi l'esegeta.

Sotto questo rispetto merita espressione, dapprincipio, il fatto che, se si accoglie il testo con due $\mu \varepsilon \theta i ́ \sigma \tau \alpha \sigma \theta \alpha$, è bensì vero che la tradizione manoscritta risulta concorde in merito ma il senso sfiora una sostanziale tautologia, più che essere una delle ridondanze che punteggiano talora il fraseggio di Aristotele: 'è necessario che (scil. il corpo che si sposta) qualora non si comprima - si sposti verso ciò verso cui si sposta per natura'; i due kola di cui si struttura il periodo, infatti, potrebbe sembrare che dicano pressoché lo stesso; in realtà, senza voler de lana caprina rixari, si deve cogliere il senso dell'opposizione tra i due membri - nonché il senso del loro incremento di senso - nel contrasto tra il senso del

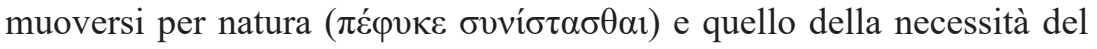

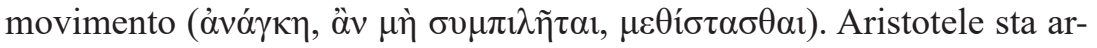
gomentando ${ }^{14}$ che, se si immette un cubo nell' acqua, l'acqua sotto il cubo

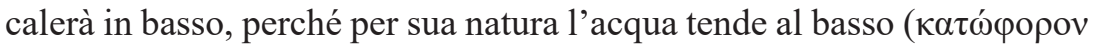

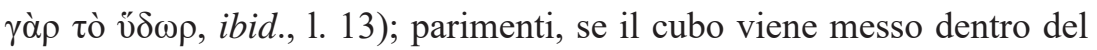
fuoco, quest'ultimo si sposterà ancora ma, questa volta, verso l'alto, perché per natura il fuoco tende all'alto (ávópopov, ibid., 1. 14); questo stato di cose non interviene in due casi, continua lo Stagirita: o nel caso in cui si consideri un movimento violento, cioè non conforme a natura (Aristotele adduce l'esempio dell'acqua in un recipiente: il cubo non potrà premere l'acqua verso il basso perché il fondo del recipiente oppone resistenza, ragione per cui l'acqua avanzerebbe verso l'alto - e non verso

14 Philop., In Phys. 664, 10-26 (ed. Vitelli). 
il basso - per far posto al cubo; lo stesso nel caso del fuoco: se non ha spazio per salire, immessovi un cubo, il fuoco si svilupperà verso il basso) o nel caso in cui si verifichi una compressione. Questa è la condi-

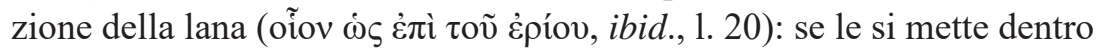
il cubo, essa non andrà né verso l'alto né verso il basso bensì si contrarrà in se stessa. Tale condizione è rilevante perché è quella considerata

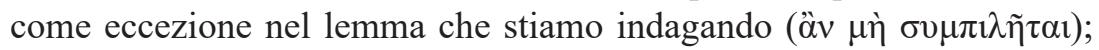
eliminata questa circostanza, il filosofo direbbe che il movimento è necessariamente verso ciò verso cui si muove per natura (venendo quindi

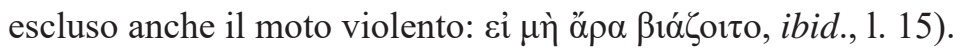

\section{INTORNO ALL'UNICITÀ DELL'ATTESTAZIONE DI FILOPONO}

Come si constata, il senso del passo è sottile sia con $\mu \varepsilon \theta i ́ \sigma \tau \alpha \sigma \theta \alpha \iota$ sia, a fortiori, con la lectio difficilior $\sigma v v i ́ \sigma \tau \alpha \sigma \theta \alpha \mathrm{r}$; ma, oltre a essere sottile, riguarda una questione tutto sommato minore, non decisiva nell'economia più generale del tema che Aristotele sta dipanando. È questo il motivo per cui da altri commentatori fuori di Filopono - come dal contemporaneo Simplicio - la questione non sia stata accampata affatto nel dettaglio, da cui deriva che non sappiamo quale verbo leggessero nel passo in esame. Rispetto a questo, Simplicio, commentando Phys.

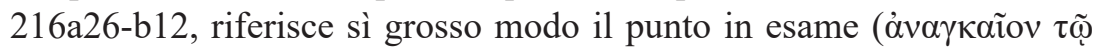

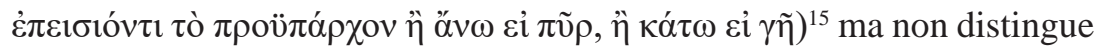
analiticamente $\mathrm{i}$ casi appena visti, la qual cosa comporta la conseguenza che non venga citata specificamente la microsezione di nostro interesse. Nulla quaestio da parte di Simplicio e, non citando nel dettaglio la lettera, non sappiamo che lezione avesse davanti ma, per certo, qualunque fosse il testo aristotelico sotto i suoi occhi, questo doveva essere fondamentalmente coerente e senza troppi problemi nel senso. Altrettanto si può dire già per un metafraste di Aristotele - più che suo commentatore - qual è Temistio ${ }^{16}$, alla metà del IV secolo; questi, in riferimento

$15 \quad$ Simpl., In Phys. 680, 33 - 681, 5 (ed. Diels).

16 Temistio non parla di parafrasi circa il suo $\sigma 0 ́ \gamma \gamma \rho \alpha \mu \mu \alpha$ (cfr. Them., Or. 23 [89, 20 - 90, 5 Downey - Norman]) bensì genericamente di $\pi \rho \alpha \gamma \mu \alpha \tau \varepsilon i ̃ \alpha ı$ come quando sta 
alla pericope 216a20-30, non va oltre alcune generali osservazioni sul punto in questione, così da ricordare che è necessario che si sposti ciò

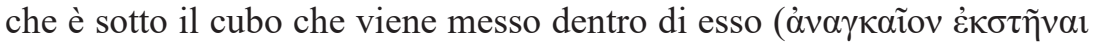

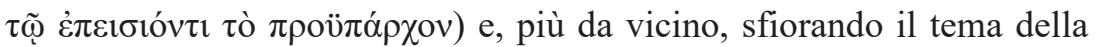

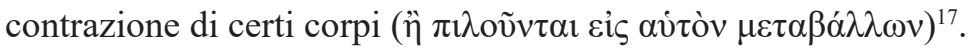

Si compulsa così un punto molto delicato: affrontando Simplicio e Temistio consta che la lezione a loro fruibile non è a noi né nota né ricavabile ma, quale che fosse il loro testo, non doveva sollevare soverchio interesse e soprattutto non doveva sollevare problemi di alcuna sorta, tanto che non ne discutono nello specifico e, nemmeno, riportano la pericope di stretta pertinenza. Un rilievo si impone: la lezione $\mu \varepsilon \theta i ́ \sigma \tau \alpha \sigma \theta \alpha$ prevale sì ma a partire dalla tradizione dei codici quando invece, in ordine a un'epoca più risalente, non sappiamo nulla se non la testimonianza di Filopono, il quale conosce soltanto $\sigma v v i ́ \sigma \tau \alpha \sigma \theta \alpha$ con il già introdotto corollario che $\mu \varepsilon \theta$ í $\tau \alpha \sigma \theta \alpha$ l è citato dall'alessandrino non già come variante ma come esplicazione di senso, essendogli pari, come abbiamo visto dire espressamente.

Si sta quindi configurando la possibilità di avere accesso a una lezione - pur minima ma rilevante - del testo di Aristotele ancora circolante a livello di inizio VI secolo, poi cassata in un processo di uniformazione della lettera, favorita dal fatto che il senso fondamentalmente non ne avrebbe sofferto, giacché lo stesso Filopono sostiene che le due lezioni sono pressoché pari. Si può valutare ora, recta uia, un aspetto già lambito, cioè in che cosa consista il carattere di difficoltà di $\sigma u v i ́ \sigma \tau \alpha \sigma \theta \alpha l^{18}$ rispetto a $\mu \varepsilon \theta i ́ \sigma \tau \alpha \sigma \theta \alpha$. Va notato, intanto, che $\sigma v v i ́ \sigma \tau \eta \mu$ è un verbo poco sfruttato da Aristotele nella Fisica: se ne registrano 8 occorrenze sulle 423 dell'intero corpus $^{19}$; là dove l'impiego non sia puramente descrittivo ma rivesta una qualche funzione teoretica, il senso non è affatto connotato da valore di moto, come invece richiederebbe 'ś $\varphi^{\prime}$ ó nella sua

commentando De an. I, 3 (è Simplicio a parlare di parafrasi in rapporto a Temistio: cfr. Simpl., De cael. 188, 30).

17 Them., In Phys. 133, 16-20 e 26-28, (ed. Wallies).

18 Può essere ricordato che questo verbo non manca di sollevare difficoltà interpretative anche altrove, come nel caso di Arist., De an. I, 2-5 (Themistius 1996: 28, n. 5).

19 Così giusta Radice, Bombacigno 2005: sub uoce. Dalla stessa fonte, sub uoce, si ricava che $\mu \varepsilon \theta i ́ \sigma \tau \eta \mu$ è verbo di maggior sfruttamento nella Fisica (10 occorrenze sulle 47 complessive). 
immediata accezione locale e come comporta anche il significato di sostanziale sinonimia con $\mu \varepsilon \theta i ́ \sigma \tau \alpha \sigma \theta \alpha$ r rilevato anche dal commentatore alessandrino.

Si considerino, a suffragio, almeno i seguenti passi di Aristotele:

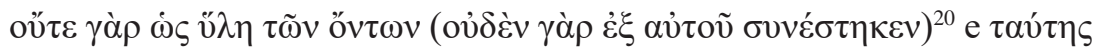

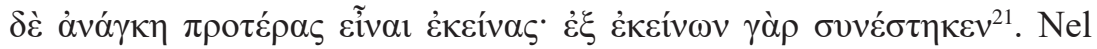
primo specimine Aristotele sta speculando sulla natura del $\tau$ ó $\pi$ o $\zeta$, rilevando che esso non è $\tilde{\imath} \lambda \eta$; per questa ragione - egli precisa - non ne consiste. Nell'altro luogo riferito, ritorna - questa volta in accezione positiva - il senso di 'consistere', circa il moto misto, come consistente sia del moto lineare sia di quello circolare. Come si constata, è questo

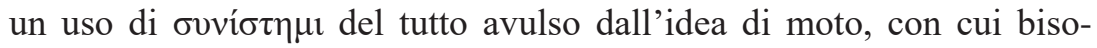
gna invece fare i conti nel nostro caso: come può essere inteso ab origine 'verso cui consiste', se è vero che qualcosa consiste di qualcos'altro e non già nello spostamento verso qualcosa? Abbiamo sottolineato 'ab origine' (cioè nella lezione aristotelica), perché in fondo lo stesso Filopono sembra, alla fine, cedere e andare nella direzione semplificatoria del senso di moto (il qual senso, come si è detto, fondamentalmente regge, ma non senza difficoltà nello specifico della lettera). Si configura una situazione netta, in cui è come se il valore di moto insito in $\dot{\varepsilon} \varphi$ ' ó avesse trascinato a considerare in accezione di moto $\sigma v v i ́ \sigma \tau \alpha \sigma \theta \alpha \mathrm{l}$ (significato non impossibile di per sé ma certo non esperito dall'usus scribendi di Aristotele), senza tuttavia che questo verbo possa essere valutato in tal senso sine glossa, tanto più che - entro il valore di movimento - si faticherebbe a capire in valore del prefisso $\sigma v v$ - (infatti, un qualche vero senso di unione a qualcos'altro non è prioritario). Si noti, da subito, che anche Filopono privilegia di gran lunga il valore ontologico e statico di consistenza circa $\sigma v v i ́ \sigma \tau \eta \mu t^{22}$, segnatamente perché indotto dal valore che gli ascriveva Aristotele - e di cui doveva tenere conto, essendo in ambito ipomnematico. Sarà il contesto filoponiano stesso a chiarire l'intera uexata quaestio; Filopono sta infatti commentando un tipo di moto molto specifico in Aristotele, quello connesso con la natura del ciò di cui si dice. Anzitutto il commentatore si pronuncia brevemente intorno al

\footnotetext{
20 Arist., Phys. 209a21.

21 Arist., Phys. 265a16.

22 Basterà rinviare a Philop., In Phys. 8, 20; 97, 3; 103, 34; 265, 1; 806, 34 (ed. Vitelli).
} 
ruolo della questione:

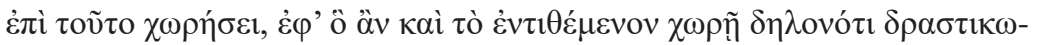

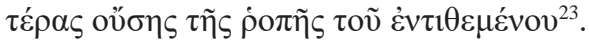

Se, quindi, si introduce un cubo dentro l'acqua, questa avanzerà verso il basso, come anche il cubo stesso, perché la natura dell'acqua tende al basso, ammesso che la po trimenti non si avrebbe alcuno spostamento. Quello che rileva osservare è che il movimento verso il basso è tale poiché legato alla natura dell'acqua: è proprio il rimando alla naturalità di questo moto a giustificare sia

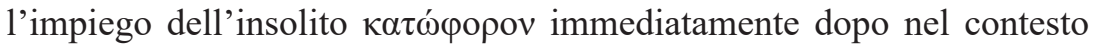

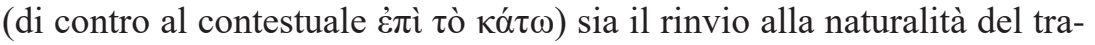
sferimento nella voce aristotelica del lemma (il $\pi \dot{\varepsilon} \varphi v \kappa \varepsilon$ di $\dot{\varepsilon} \varphi$ ' ô $\pi \varepsilon ́ \varphi v \kappa \varepsilon$ $\sigma v v i ́ \sigma \tau \alpha \sigma \theta \alpha$ ) $)$. A ruoli invertiti, se il cubo è posto nel fuoco, il movimento del cubo sarà sempre verso il basso ma il fuoco si alzerà verso

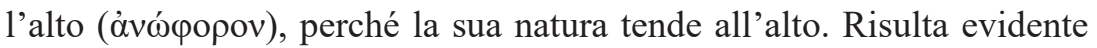

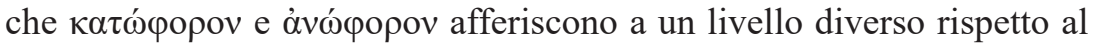

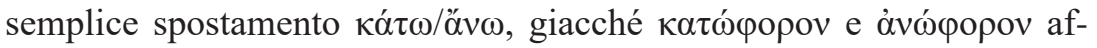
feriscono alla natura dell'acqua e del fuoco ${ }^{24}$, prima ancora che al movimento cui pur dànno luogo. Si tratta di una accezione, quindi, che rimanda sì a uno spostamento ma il moto è da intendere - per dire così - in senso secondario, visto che in senso primario riguarda la natura di ciò di cui si predica, prima ancora che il moto medesimo sia messo in atto.

In questo modo cambia la prospettiva dalla quale rileggere la questione; si tratta, infatti, di aver che fare con un rispetto statico del moto, in quanto pertinente alla natura del corpo, prima che al suo effettivo spostamento. Siccome un corpo è - rectius: è e consiste - della sua propria natura, si comprenderà facilmente che la lezione da cui siamo partiti è

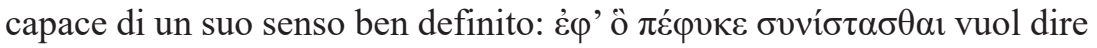
che il corpo, consistendo di una certa natura ed essendo proprio di una certa natura avere un certo spostamento, si sposterà in quella direzione.

\footnotetext{
23 Philop., In Phys. 665, 6-7 (ed. Vitelli).

24 Sul valore marcato di questa terminologia in riferimento a un movimento legato alla natura del mobile, Filopono si è già pronunciato in contesto prossimo, commentando ad es. Phys. $216 a 26$ e 217 a19 passim.
} 
In altri termini, interviene qui una espressione brachilogica, come tipico peraltro di Aristotele, per indicare che il corpo in cui è posto il cubo ha una certa natura e, in forza di essa, il medesimo corpo si sposterà, una volta introdotto il cubo. Ecco allora che si potrà intendere 'verso cui risulta per natura consistere' come la conflazione di due segmenti di senso, cioè 'verso cui la sua natura tende' e 'risulta consistere per natura' (ovvero ha una certa natura e quella natura ha, tra le altre proprietà, quella di muoversi nella direzione detta).

Filopono lascia un passo nitido in cui dimostra di connettere il tipo del movimento a ciò di cui il corpo consiste ( $\sigma$ $\sigma \tau \alpha \sigma ı)$ ) allorché osserva, commentando Phys. 686, 5-9 (ed. Vitelli), che

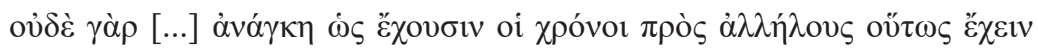

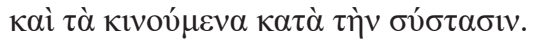

Filopono sta rimarcando che, dati due corpi disomogenei tra loro, non si dà alcuna relazione tra i medesimi; ciascuno di essi si muove come compete alla natura di cui consiste e che conferisce loro la consistenza che li sostanzia; purtuttavia si può istituire una relazione tra i tempi dei movimenti rispettivi. A noi interessa la prima parte dell'argomento: i corpi sono disomogenei e quindi si muovono in modo tra loro diverso, perché diversa è la loro consistenza: basterà questo per introdurre la relazione tra consistenza e moto che ne deriva. Quanto osservato induce quindi a pensare che, nel passo che stiamo esaminando, Aristotele trattasse del movimento che è potenzialmente connesso all'oggetto, in quanto legato alla natura del corpo di cui si dice; in forza di ciò, si può portare un ultimo corollario: il moto così inteso in senso potenziale e pressoché statico afferisce più al 'come' che al 'dove' o, propriamente, attraverso la sua direzione, tale moto permette di capire in che cosa consista il corpo in indagine, la sua natura ${ }^{25}$. Si restituirà così al passo il

25 Di questa accezione del moto in riferimento al 'come' è esplicita testimonianza un passo della Fisica aristotelica ampiamente considerato da Simplicio (212b2 ss.) quando

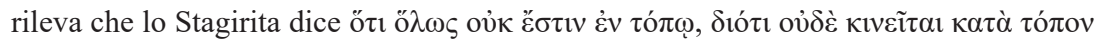

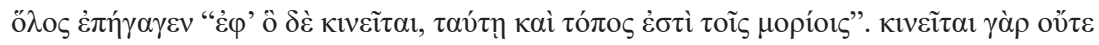

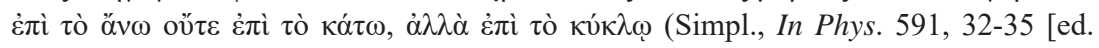
Diels]; circa la posizione di Massimo di Efeso, cui si richiama in merito, cfr. ibid. 592, 6-8). Simplicio permette di cogliere ulteriormente la problematicità di intendere '̇ं $\varphi^{\prime}$ 
seguente senso: 'è necessario che si sposti verso ciò verso cui la natura di cui consiste tende'.

\section{VERSO UNA CONCLUSIONE}

Il caso da noi indagato è solo uno spiraglio, altrettanto limitato che rilevante per la sua capacità di far tralucere qualcosa del lesto di Aristotele avanti il VI secolo; come osserva Henri Carteron ${ }^{26}$ (ibid., pp. 6-7), infatti, la nostra tradizione manoscritta della Fisica pare rimontare a un archetipo comune posteriore al VI e anteriore al IX (come consta dalle interpolazioni in Averroè), quando invece prima del VI dovevano circolare lezioni divergenti - rilevante ma ignoto è quale ruolo abbia avuto nella loro genesi la revisione portata alla Fisica da Eudemo, di cui di rado ci è pervenuta tangenzialmente una flebile eco, come nel caso di Aspasio circa $220 \mathrm{~b} 4$ (con dissenso di Temistio) e la strana lezione di 219b8 rispetto a Simpl. 716, 33 (ed. Diels).

Una rondine non fa primavera e il caso preso in esame né vuole prestarsi a generalizzazioni circa il valore delle lezioni nei commentatori neoplatonici rispetto ai testimoni medievali né - ancor meno - vuole sostituire alla lezione invalsa la variante filoponiana nella nostra fattispecie. Si ambisce - e questo verrà rivendicato con forza - l'importanza

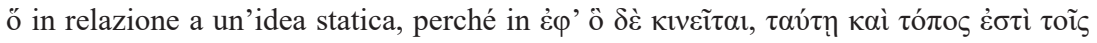

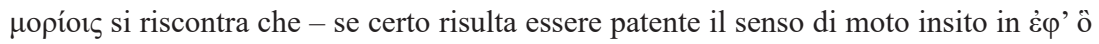
$\delta \varepsilon \grave{\varepsilon} \kappa ı v \varepsilon i \tau \tau \iota 1$ - non manca tuttavia di essere singolare la ripresa mediante $\tau \alpha$ v́t col suo valore statico: perché Aristotele dovrebbe dire che, quanto alle parti, il luogo è 'così', dopo aver detto della direzione (il 'dove') in cui si muove? In realtà, il senso di

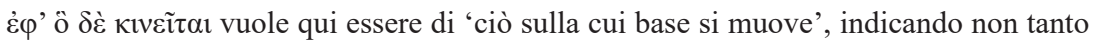
la direzione ma la natura sulla cui base il corpo in indagine si muove (e, secondaria-

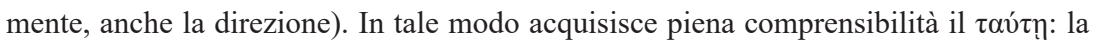
relazione tra il luogo e le parti si regola sulla base del tipo di movimento coinvolto (si noti che, proprio in quest'ottica, Simplicio chiosa dicendo che in questo caso si tratta di

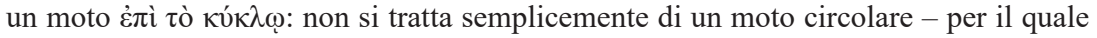

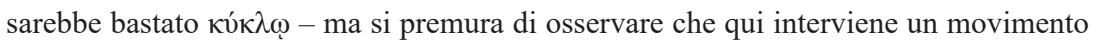
'sulla base del cerchio', poiché la direzione del moto rispecchia un livello più profondo, quello della consistenza e dell'ontologia del corpo, da cui anche la direzione e il tipo del movimento dipendono).

26 Nell'introduzione ad Arist., Physique (I-IV), op. cit., 6-7. 
di valutare specificamente varianti che, come quella in oggetto, vantano un'indubbia antichità e non possono essere nate per una mera banalizzazione del testo.

Così il caso che la presente, breve nota ha voluto accostare pone innanzi a un dato di fatto: nel caso in esame Filopono ci offre una possibilità preziosa di affacciarci a una forma testuale aristotelica che - al di là di giudizi di merito - precede la pur ancora fluida stagione medievale della Fisica; Filopono poteva leggere evidentemente un testo diverso da quello che la tradizione canonizzerà e con questo fatto i moderni sono chiamati almeno a confrontarsi, cercando una contestualizzazione del fenomeno che tenga conto sia della perizia filologica del commentatore antico sia della sua acribia speculativa. Forse anche oltre le sue intenzioni, la perizia di Filopono ha qui salvato una variante del testo aristotelico per il resto del tutto naufragata nel tempo; da ciò non è lecito domandare più di quello che possa restituire, se non cioè una testimonianza - minuta ma localmente precisa - circa la fluttuazione del testo di Aristotele ancora viva sotto i commentatori neoplatonici (e su cui gli stessi potevano o dovevano ancora intervenire). Pur restringendone il raggio d'azione a quel poco che da un singolo caso si può trarre, non si potrà allora che concordare con Carteron quando scrive, circa il ruolo della tradizione indiretta dei commentatori: «l'importance que présente, pour l'établissement du texte de la Physique et de son histoire, l'étude des commentateurs ne saurait être exagèrée»» ${ }^{27}$.

\section{BIBLIOGRAFIA}

\section{Fonti}

Aristote, 1990 (19261), Physique (I-IV). Texte établi et traduit par H. Carteron, vol. I, Paris.

Philoponus I., 2012, On Aristotle Physics 4, cap. 6-9, trad. di P. Huby, London.

Ross W. D., 1979 (1936 $)$, Aristotle's Physics: A Revised Text with Introduction and Commentary, Oxford.

Themistius, 1996, On Aristotle, On the Soul, trad. di R. B. Todd, London. http:// dx.doi.org/10.5040/9781472552440.

$27 \quad$ Nell'introduzione ad Arist, Physique (I-IV), op. cit., 5-6. 
Vitelli G. (ed.), 1888, Ioannis Philoponi in Aristotelis Physicorum libros quinque posteriores commentaria, Berolini. https://doi.org/10.1515/9783111435411.

\section{Studi}

Algra K. A., 1995, Concepts of Space in Greek Thought, Leiden. https://doi. org/10.1163/9789004320871.

Bonitz H., 1870, 'Index Aristotelicus', [in:] Aristotelis Opera, ed. Academia Regia Borussica, vol. V, Berolini.

Crisci E., Degni P. (eds.), 2011, La scrittura greca dall'antichità all'epoca della stampa. Una introduzione, Roma.

Diels H., 1882, Zur Textgeschichte der Aristotelischen Physik, Berlin.

Golitsis P., 2008, Les Commentaires de Simplicius et Jean Philopon à la Physique d'Aristote. Tradition et innovation, Berlin-New York. https://doi. org/10.1515/9783110208078.

Kühner R., Gerth B., 1904, Ausführliche Grammatik der griechischen Sprache. Satzlehre, vol. II, Hannover-Leipzig.

Messeri G., Pintaudi R., 2000, I papiri greci d'Egitto e la minuscola libraria, [in:] I manoscritti greci tra riflessione e dibattito. Atti del V Colloquio Internazionale di Paleografia Greca (Cremona, 4-10 ottobre 1998), G. Prato (a cura di), vol. I-II, Firenze, pp. 67-82.

Radice R., Bombacigno R. (eds.), 2005, Aristoteles (Lexicon III), vol. I-II, Milano.

Rashed M., 2008, Alexandre d'Aphrodise, Commentaire perdu à la Physique d'Aristote (Livres IV-VIII): Les scholies byzantines. Édition, traduction et commentaire, Berlin-New York.

Rashed M., 2016, L'Héritage aristotélicien: Textes inédits de l'Antiquité. Nouvelle édition revue et augmentée, Paris.

Rescigno A., 2017, 'Nuovi frammenti del Commento di Filipono ai libri V-VIII della Fisica', Studia graeco-arabica 7, pp. 75-104.

Schwyzer E., 1950, Griechische Grammatik auf der Grundlage von Karl Brugmanns Griechischer Grammatik, vol. II (Syntax und Syntaktische Stilistik), München.

Sjoerd Hasper P., 2020, The Greek Manuscripts of Aristotle's Physics, [in:] Aristotle's Physics VIII, Translated into Arabic by Ishaq ibn Hunayn $\left(9^{\text {th }}\right.$ c.). Introduction, Edition, and Glossaries, R. Arnzen (ed.), Berlin-Boston, pp. CXIII-CLXXXVII, https://doi.org/10.1515/9783110582086. 